\title{
Myocardial Consumption of Oxygen and Carbohydrates in Newborn Sheep
}

\author{
DAVID J. FISHER, ${ }^{(22)}$ MICHAEL A. HEYMANN, AND ABRAHAM M. RUDOLPH \\ Cardiovascular Research Institute and the Departments of Pediatrics, Physiology, and Obstetrics, Gynecology and \\ Reproductive Sciences, University of California, San Francisco, California, USA
}

\begin{abstract}
Summary
We measured blood flow to the myocardium of the left ventricular free wall, and blood glucose, lactate, pyruvate, and oxygen concentrations simultaneously in the aorta and coronary sinus 13 times in seven previously instrumented newborn sheep, 4 to 25 days after birth. We calculated arteriovenous difference and consumption of oxygen, glucose, lactate, and pyruvate by the newborn myocardium. Results were compared with recently obtained measurements in the myocardium of fetal and adult sheep (6).

Myocardial consumption of oxygen in the newborn $(577 \pm 38$ $\mu \mathrm{M} \cdot \min ^{-1} \bullet 100 \mathrm{~g} \mathrm{LV}^{-1}$ ) was higher than in either the fetuses or the adults. This was associated with a greater myocardial blood flow $\left(201 \pm 21 \mathrm{~mm} \cdot \mathrm{min}^{-1} \cdot 100 \mathrm{~g} \mathrm{LV}^{-1}\right)$ in the newborns. However, the increased myocardial oxygen consumption in the newborns was commensurate with their increased cardiac work as compared with both the fetuses and adults.

Although there is an abrupt postnatal increase in arterial glucose concentration, there was no significant difference in either the myocardial consumption of glucose or the contribution of glucose to the total myocardial energy supply among fetal, newborn or adult sheep. Postnatal decreases in myocardial consumption of lactate and pyruvate are not compensated for by an increase in glucose consumption. In newborn sheep, carbohydrates including glucose, lactate, and pyruvate supply the substrate for no more than approximately one-fourth of the total myocardial energy demands (carbohydrate/oxygen quotient was 0.26 ).
\end{abstract}

\section{Speculation}

Although glucose and lactate supply most of the substrate for myocardial energy supply in the lamb fetus, postnatally there is an abrupt decrease in lactate utilization that is not compensated for by an increase in glucose utilization. The identity of the primary myocardial energy substrate(s) in newborn and adult sheep remains to be determined.

Breuer et al. (2) have suggested that myocardial metabolism is primarily dependent on glucose in the neonatal period. Recently, we have demonstrated that glucose alone could provide the substrate for no more than one-third of the myocardial energy demands of fetal or adult sheep (7). Furthermore, we have shown that lactate may supply the substrate for up to $60 \%$ of total fetal myocardial energy demands (7). To determine if the myocardium of a newborn animal is primarily glucose dependent or, in general, carbohydrate dependent, we measured the myocardial consumption of oxygen, glucose, lactate, and pyruvate in conscious newborn lambs.

\section{MATERIALS AND METHODS}

\section{SURGICAL TECHNIQUE}

Using aseptic technique, we operated on seven Western newborn lambs 1 to 17 ( $9 \pm 2$, mean \pm S.E. $)$ days after birth. We anesthetized the lambs with 0.5 to $1 \%$ halothane, intubated the trachea, and ventilated the lungs with a Harvard pump. Through a thoracotomy in the left fourth intercostal space, we inserted polyvinyl catheters (inside diameter, $0.076 \mathrm{~cm}$; outside diameter, $0.122 \mathrm{~cm}$ ) into the left atrium through a purse-string suture in the atrial appendage, the aorta via the left internal thoracic artery, the left internal thoracic vein, and the coronary sinus (7).

We retracted the left lung and exposed the hemiazygous vein. In sheep, this vein regularly joins the great cardiac vein to form the origin of the coronary sinus (12). We ligated the hemiazygous vein distally, inserted a polyvinyl catheter (insider diameter, 0.076 $\mathrm{cm}$; outside diameter, $0.122 \mathrm{~cm}$ ), and advanced it 1 to $1.5 \mathrm{~cm}$ so that the catheter tip was located at the origin of the coronary sinus. We filled all of the catheters with heparin (1000 units/ml) and sutured the wounds. We exteriorized the catheters on the left side of the chest and protected them with a Teflon cloth pouch sewn to the skin.

We gave potassium penicillin $\mathrm{G}$ (one million units) and kanamycin sulfate $(15 \mathrm{mg} / \mathrm{kg})$ intravenously on the day of surgery and on the first postoperative day. We drained the heparin from the catheters daily and refilled them with fresh heparin.

\section{EXPERIMENTAL PROTOCOL}

The lambs were allowed to feed from the ewes until one hour prior to a study. At that time they were placed in a canvas sling and we began a continuous recording of arterial blood pressure and heart rate. After the lambs had been resting quietly for 30 min, we withdrew $5 \mathrm{ml}$ blood samples simultaneously from the aorta and coronary sinus and measured myocardial blood flow. On each sample we measured blood glucose, lactate, and pyruvate concentrations, oxygen content, hematocrit, hemoglobin, $\mathrm{PO}_{2}, \mathrm{pH}$, and $\mathrm{PCO}_{2}$. Immediately after withdrawing the blood, we injected $15 \mu$ diameter radionuclide-labeled microspheres into the left atrium while withdrawing a reference sample from the ascending aorta (7).

One to three studies were performed in each of the seven lambs 3 to $10(5 \pm 0.6$, mean \pm S.E. $)$ days after surgery. Repeat studies in individual lambs were performed at least 2 days apart. A total of 13 studies were performed. The ages of the lambs on the study days ranged from 4 to 25 days.

\section{MEASUREMENTS AND CALCULATIONS}

We have described the methods we used to measure blood glucose, lactate, and pyruvate concentrations; oxygen content; blood flow to the left ventricular free wall; $\mathrm{PO}_{2} ; \mathrm{pH} ; \mathrm{PCO}_{2}$; heart rate, and aortic blood pressure in detail previously (7). Samples for measurement of glucose, lactate, and pyruvate concentrations were deproteinized immediately. Blood glucose was measured in duplicate by the glucose oxidase method, and blood lactate and pyruvate were measured in duplicate by the lactic dehydrogenase method (Sigma Chemical Company, St. Louis, MO). Blood oxygen content was measured in duplicate directly (Lex- $\mathrm{O}_{2}$-Con; Lexington Instrument Corporation, Waltham, MA). The mean 
percentage of error for paired samples was less than $2 \%$ for blood glucose, lactate, and oxygen concentrations and less than $4 \%$ for blood pyruvate concentration.

Myocardial blood flow to the left ventricular free wall was measured by the radionuclide-labeled microsphere technique. Each measurement was done with $15 \mu$ diameter microspheres labeled with one of the following radionuclides: ${ }^{125} \mathrm{I},{ }^{141} \mathrm{Ce},{ }^{51} \mathrm{Cr}$, ${ }^{85} \mathrm{Sr},{ }^{95} \mathrm{Nb}$, or ${ }^{46} \mathrm{Sc}$. During each injection of the microspheres into the left atrium, a reference sample was withdrawn continuously from the ascending aorta into a preweighed syringe for either 1 or $1.25 \mathrm{~min}$ at a rate of 4 or $7 \mathrm{ml} / \mathrm{min}$. After separation from the remaining myocardium, the left ventricular free wall was weighed (Mettler P1200; Mettler Instrument Corp., Highstown, NJ) and incinerated. The radioactivity of the left ventricular free wall was measured in a 512-channel pulse height analyzer (Searle Analytical, Des Plaines, IL), and blood flow to the left ventricular free wall was calculated as described previously $(7,8)$. Each portion of myocardium and each reference sample had at least 1600 microspheres in it (8).

Arterovenous difference (AV) across the left ventricular free wall for each substrate was the difference between the measured concentrations in blood obtained from the ascending aorta and coronary sinus. Because we sampled blood from the coronary sinus, we wanted to measure blood flow to that part of the myocardium drained by the coronary sinus. Although the coronary sinus of newborn lambs receives small amounts of blood flow from the left atrium and the right ventricular free wall, approximately $85 \%$ of coronary sinus flow comes from the left ventricular free wall and ventricular septum (5). Because blood flows to the left ventricular free wall and septum are similar (6) in newborn lambs, we estimated left ventricular myocardial consumption of each substrate by the product of arteriovenous difference of the substrate across the left ventricular free wall and blood flow to the left ventricular free wall.

We estimated the percentage of total left ventricular myocardial oxidative metabolism that could be accounted for by comlete combustion of a substrate by calculating substrate/oxygen quotients for glucose, lactate, and pyruvate using the following formula:
Substrate/oxygen quotient $=\frac{\mathrm{AV} \text { substrate }}{\mathrm{AV} \text { xygen }} \times$ factor

The factors are: glucose, 6 ; lactate, 3 ; and pyruvate, $2.5(1,4,16)$. The factors are derived from the stoichiometric relationships between oxygen and the respective carbohydrates when expressed in equivalent molar concentrations.

The mean \pm standard error was calculated for each variable. The results were compared with our previously published values for fetal and adult sheep (7) by means of analysis of variance. When the computed $F$ ratio exceeded the critical $F$ ratio (at $P=$ $0.05)$, the Newman-Keuls test was used to identify the group(s) that were significantly different $(19,21)$. In addition, we performed linear regression analysis of the data to determine if there was any significant change in the variables during the neonatal period (19).

\section{RESULTS}

The blood gases and $\mathrm{pH}$ in systemic arterial blood were within normal limits compared to measurements made in our laboratory in unanesthetized, previously instrumented lambs of similar postnatal age (10). $\mathrm{Po}_{2}$ was $75 \pm 3$ torr, $\mathrm{PCO}_{2}$ was $40 \pm 1$ torr, and $\mathrm{pH}$ was $7.41 \pm 0.01$. Arterial blood hematocrit was $27 \pm 2 \%$, and hemoglobin concentrations were $9.0 \pm 0.8 \mathrm{~g} / \mathrm{dl}$. Systolic, diastolic, and mean arterial blood pressures were $101 \pm 3,66 \pm 2$, and 79 $\pm 2 \mathrm{~mm} \mathrm{Hg}$, respectively, and heart rate was $214 \pm 3$ beats $/ \mathrm{min}$. These values were all in the normal range. The rate-pressure product was $21772 \pm 1669$ beats $\cdot \mathrm{mm} \mathrm{Hg} \cdot \mathrm{min}^{-1}$ (8).

Blood flow to the free wall of the left ventricle in the newborn was $201 \pm 21 \mu \mathrm{M} \cdot \mathrm{min}^{-1} \cdot 100 \mathrm{~g} \mathrm{LV}^{-1}$ as compared with our previously obtained results of $156 \pm 11 \mathrm{ml} \cdot \mathrm{min}^{-1} \cdot 100 \mathrm{~g} \mathrm{LV}^{-1}$ in the 120 day gestation lamb fetus and $82 \pm 8 \mathrm{ml} \cdot \mathrm{min}^{-1} \cdot 100 \mathrm{~g} \mathrm{LV}^{-1}$ in adult sheep (7). There was no change in the left ventricular myocardial flow per $100 \mathrm{~g}$ of left ventricle during the 25 days after birth, the period when we made observations.

Oxygen content was $4243 \pm 377 \mu \mathrm{M} \cdot 1^{-1}$ in aortic blood and $1128 \pm 161 \mu \mathrm{M} \cdot 1^{-1}$ in the coronary sinus blood, and the arteriovenous difference of oxygen across the myocardium was $3115 \pm$ $277 \mu \mathrm{M} \cdot 1^{-1}$ (Fig. 1). Oxygen consumption by the left ventricular myocardium was $577 \pm 38 \mu \mathrm{M} \cdot \mathrm{min}^{-1} \cdot 100 \mathrm{~g} \mathrm{LV}^{-1}$, and it did not

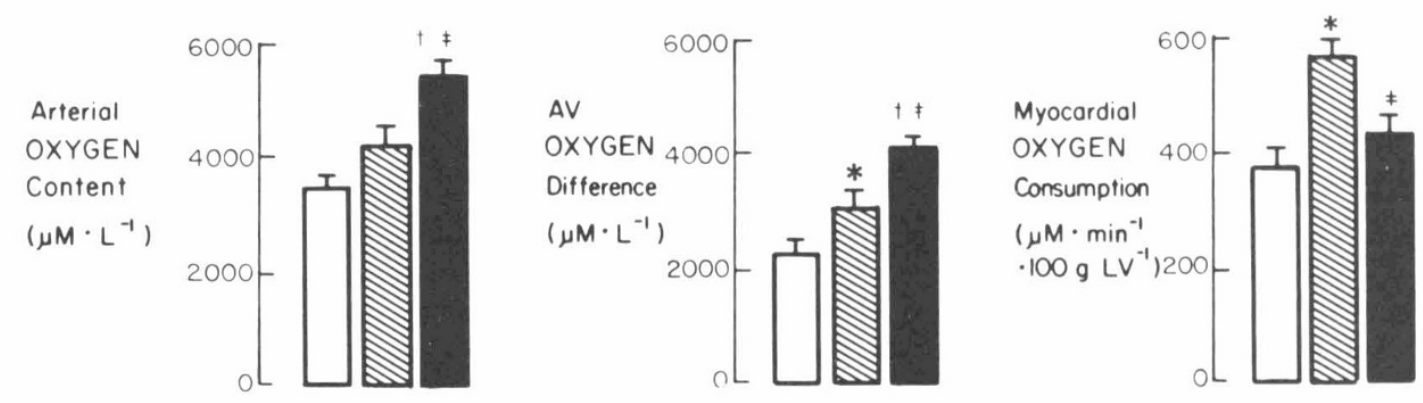

Fig. 1. Arterial oxygen content, AV difference of oxygen across the left ventricular myocardium, and consumption of oxygen by the myocardium of the left ventricular free wall in fetal $(\square)$, newborn $(\square)$, and adult $(\mathbf{\square})$ sheep. Mean \pm S.E. ${ }^{*}$, fetuses are significantly $(P<0.05)$ different from newborns. $\dagger$, fetuses are significantly different from adults; $\neq$, newborns are significantly different from adults. Fetal and adult sheep data are from Ref. 6 .

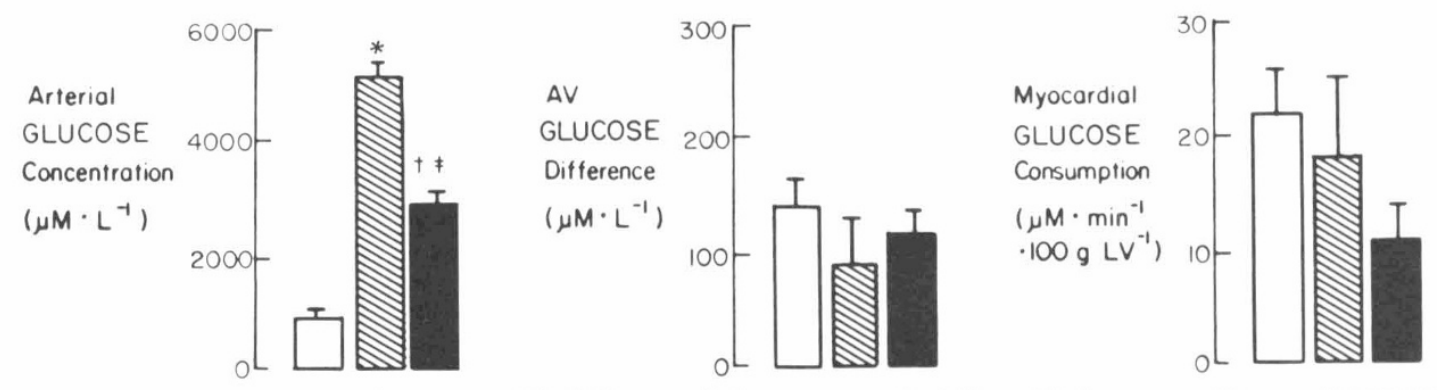

Fig. 2. Arterial glucose concentration, arteriovenous (AV) difference of glucose across the left ventricular myocardium, and consumption of glucose by the myocardium of the left ventricular free wall in fetal $(\square)$, newborn $(\square)$ and adult sheep $(\square)$. Mean \pm S.E. ${ }^{*}$, fetuses significantly $(P<0.05)$ different from newborns; $\uparrow$, fetuses significantly different from adults; $\neq$, newborns significantly different from adults. Fetal and adult sheep data are from Ref. 6. 


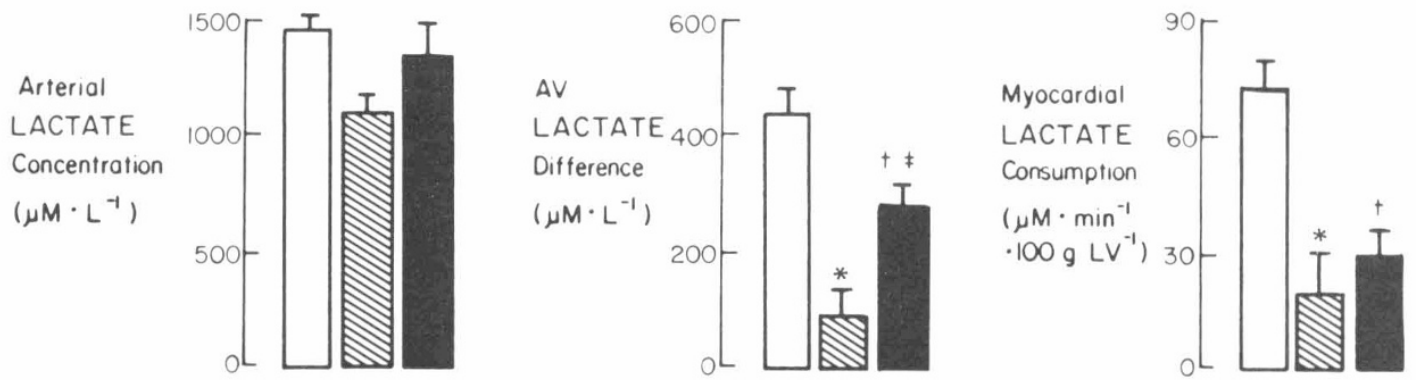

Fig. 3. Arterial lactate concentration, arteriovenous (AV) difference of lactate across the left ventricular myocardium, and consumption of lactate by the myocardium of the left ventricular free wall in fetal $(\square)$, newborn $(\square)$ and adult sheep $(\mathbf{\square})$. Mean \pm S.E. ${ }^{*}$, fetuses are significantly $(P<0.05)$ different from newborns; $\dagger$, fetuses significantly different from adults; $\ddagger$, newborns significantly different from adults. Fetal and adult data are from Ref 6 .

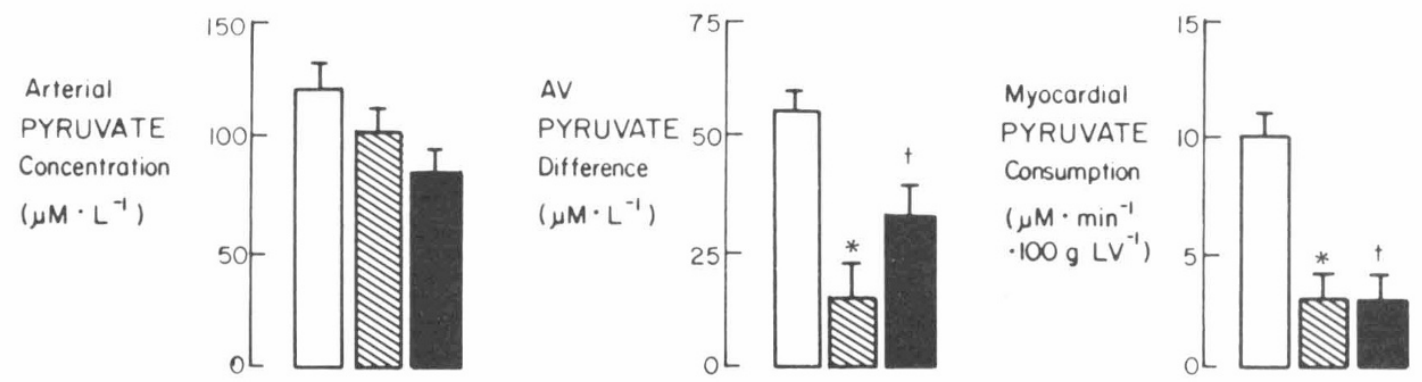

Fig. 4. Arterial pyruvate concentration, arteriovenous (AV) difference of pyruvate across the left ventricular myocardium, and consumption of pyruvate by the myocardium of the left ventricular free wall in fetal ( $\square$ ), newborn ( $\square$ ), and adult sheep (耳). Mean \pm S.E. *, fetuses are significantly ( $P$ $<0.05)$ different from newborns. $\dagger$, fetuses are significantly different from adults. $\ddagger$, indicates newborns are significantly different from adults. Fetal and adult sheep data are from Ref. 6.

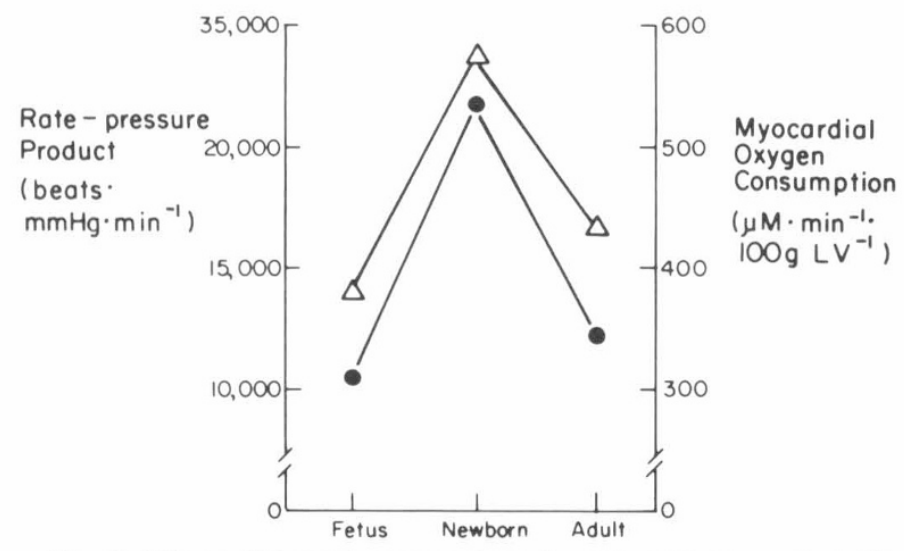

Fig. 5. Myocardial work expressed as the rate-pressure product compared with oxygen consumption $(\triangle)$ in sheep fetuses, newborns, and adults. Fetal and adult data are from Ref. 6.

change during the postnatal period that we studied.

Glucose concentration was $4562 \pm 237 \mu \mathrm{M} \cdot 1^{-1}$ in the arterial blood and $4473 \pm 218 \mu \mathrm{M} \cdot 1^{-1}$ in the coronary sinus blood. Arteriovenous difference of glucose was $89 \pm 40 \mu \mathrm{M} \cdot 1^{-1}$, and left ventricular myocardial glucose consumption was $18 \pm 9 \mu \mathrm{M}$. $\min ^{-1} \cdot 100 \mathrm{~g} \mathrm{LV}^{-1}$ (Fig. 2). Arterial and coronary sinus blood lactate concentrations were $1096 \pm 79$ and $1008 \pm 99 \mu \mathrm{M} \cdot 1^{-1}$, respectively, with an arteriovenous lactate difference of $88 \pm 38$ $\mu \mathrm{M} \cdot 1^{-1}$ and a left ventricular myocardial lactate consumption of $20 \pm 9 \mu \mathrm{M} \cdot \mathrm{min}^{-1} \cdot 100 \mathrm{~g} \mathrm{LV}^{-1}$ (Fig. 3). Pyruvate concentration in arterial blood was $102 \pm 10 \mu \mathrm{M} \cdot 1^{-1}$, and in the coronary sinus blood it was $87 \pm 10 \mu \mathrm{M} \cdot 1^{-1}$, with an arteriovenous pyruvate difference of $15 \pm 5 \mu \mathrm{M} \cdot 1^{-1}$. Left ventricular myocardial pyruvate consumption was $3 \pm 1 \mu \mathrm{M} \cdot \mathrm{min}^{-1} \cdot 100 \mathrm{~g} \mathrm{LV}^{-1}$ (Fig. 4). None of the above variables changed during the 25 days after birth when the measurements were made.

The calculated substrate oxygen quotients were glucose, 0.17; lactate, 0.08; and pyruvate, 0.01. These quotients also did not change during the neonatal period.

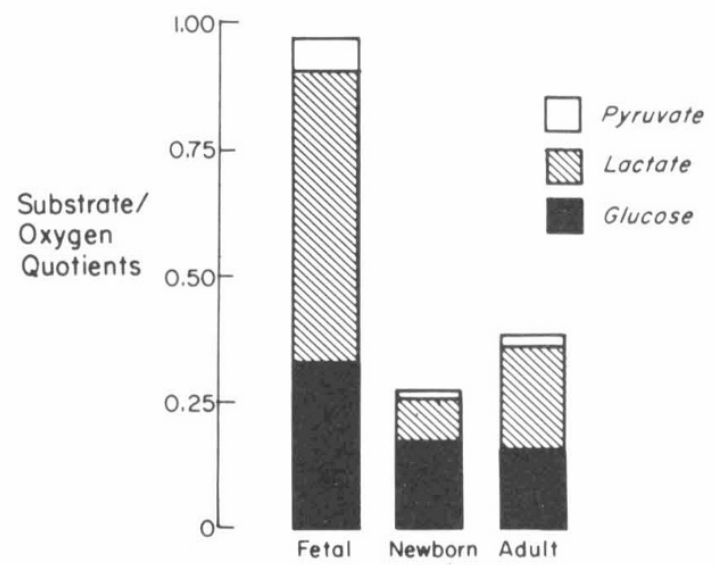

Fig. 6. Substrate/oxygen quotients for glucose (-), lactate ( $\square$ ), and pyruvate $(\square)$ in fetal, newborn, and adult sheep. Fetal and adult sheep data are from Ref. 6.

\section{DISCUSSION}

We have previously shown (7) that myocardial oxygen consumption per $100 \mathrm{~g}$ of left ventricular free wall is similar in fetal and adult sheep (Fig. 1). Cardiac work, as estimated by the ratepressure product (9), is also similar in the two groups (Fig. 5). Consequently, although arterial blood perfusing the fetal myocardium has a much lower $\mathrm{PO}_{2}(21$ to 24 torr) than in the adult, the utilization of oxygen per gram of muscle by fetal and adult myocardium should be similar (7). Oxygen consumption per 100 $\mathrm{g}$ of left ventricle is greater in the newborn than in fetal or adult sheep (Fig. 1). Because cardiac work is also greater in newborns (Fig. 5), these data demonstrate that over a wide range of arterial blood $\mathrm{PO}_{2}$ (21 to 89 torr), oxygen saturation (65 to $95 \%$ ), and oxygen content ( 2.5 to $6.5 \mathrm{mM}$ ), left ventricular myocardial oxygen consumption is determined by the myocardial work load, not by the availability of oxygen. Our results are consistent with data obtained from isolated papillary muscles, isolated intact heart preparations, and studies in adult humans and dogs in vivo (14). 
The increase in left ventricular myocardial oxygen consumption after birth and subsequent decrease in the adult parallels the changes in total body oxygen consumption and cardiac output related to weight $(10,11)$. The postnatal rise in cardiac output can be explained partly by the necessity for increasing tissue blood flow to supply the increased oxygen requirement and partly by the lower $\mathrm{P}_{50}$ of fetal blood (11). The high levels of fetal hemoglobin will have the effect of reducing the amount of oxygen extracted from blood at the $\mathrm{PO}_{2}$ levels present in arterial and venous blood postnatally (11). The significantly lower arteriovenous oxygen difference across the left ventricular myocardium in the newborn lamb is probably related to the high levels of fetal hemoglobin that are present. Thus, as in the newborn body generally, a significantly higher blood flow per unit mass is required for adequate myocardial oxygen supply.

Because they found a positive uptake of glucose, but not of lactate or free fatty acids in 7-to 12-day-old open-chested puppies, Breuer et al. (2) have suggested that glucose is the main energetic fuel of heart muscle in the early postnatal period. Based on their data (Table II) the glucose/oxygen quotient during this period is 1.10. In contrast, our data demonstrate that the glucose/oxygen quotient of previously instrumented 4- to 25-day-old sheep was 0.17 ; there was no difference in the animals studied during the first 2 wk after birth. Consequently, although glucose is an important substrate for the myocardium of sheep, glucose is not the main energetic fuel at anytime from 0.8 of gestation in the fetus through adult life (Fig. 6). It is not possible to determine if the two groups of data demonstrate species variation or occur because of differences in experimental design.

It has been suggested $(13,15)$ that the fetal heart is more dependent than the adult heart on energy supplied by glycolysis. In our study groups, myocardial glucose consumption should closely reflect the total myocardial glycolytic activity because in the steady state there should be no change in myocardial glycogen concentration. Because our data demonstrated a similar myocardial glucose consumption in fetal, newborn, and adult sheep (Fig. 2 ), we believe that myocardial glycolytic activity is also similar during these periods. In contrast, at least in sheep it appears that the major change in myocardial energy metabolism at birth is a shift away from a significant consumption of lactate.

Breuer et al. (2) have suggested that myocardial consumption of glucose is higher in the newborn than in the adult dog because arterial glucose concentration is higher in the puppy. However, they measured only arteriovenous difference of oxygen and carbohydrates across the myocardium; because they did not measure myocardial blood flow, they could not determine the actual myocardial glucose consumption. Our measurements, which included both arteriovenous difference and myocardial blood flow, show that although arterial glucose concentration was higher in the newborns (Fig. 2), there was no significant difference among the myocardial glucose consumptions of fetal, newborn, and adult sheep (Fig. 2).

We have shown previously that lactate consumption could contribute up to $60 \%$ of the substrate needed to meet total myocardial energy demands in the 0.8 gestation fetal lamb but that lactate was a relatively unimportant myocardial energy substrate in adult sheep (7). Because we have shown that lactate is also a small source for myocardial energy in newborn lambs as early as 4 days after birth, myocardial lactate consumption must decrease either late during gestation or soon after birth. It is likely that lactate ceases to be an important energy substrate for the fetal myocardium after birth when a significant exogenous source of lactate production for the fetus, the placenta (4), is abruptly removed from the circulation.

Analysis of combined substrate/oxygen quotients (Fig. 6) for glucose and lactate indicates that although nearly all of the fetal myocardial energy demands could be met by complete oxidative combustion of the amounts of glucose and lactate that are consumed, no more than one-third of newborn or adult myocardial energy demands could be met by utilization of these carbohydrates. Consequently, there must be a shift toward utilization of other substrates after birth. Because free fatty acid mobilization and utilization is closely related to survival of lambs after birth (17) and free fatty acids are known to be the primary energy substrate in adult humans and dogs (20), it is possible that free fatty acids become a major source of myocardial energy soon after birth. However, significant myocardial free fatty acid uptake was not demonstrated in newborn dogs (3) or in newborn rats (18). Thus the major myocardial energy substrate in newborn and adult sheep remains to be identified.

\section{REFERENCES AND NOTES}

I. Bing, R. J., Siegel, A., Vitale, A., Balboni, F., Sparks, E., Taeschler, M., Klapper. M., and Edwards, S.: Metabolic studies on the human heart in vivo. I. Studies on carbohydrate metabolism of the human heart. Am. J. Med., 15: 284 (1953).

2. Breuer, E., Barta, E., Pappova, E., and Zlatos, L.: Developmental changes of myocardial metabolism. I. Peculiarities of cardiac carbohydrate metabolism in the early postnatal period in dogs. Biol. Neonat., 11: 367 (1967).

3. Breuer, E., Barta, E., Zlatos, L., and Pappova, E.: Developmental changes in myocardial metabolism. II. Myocardial metabolism of free fatty acids in the early postnatal period in dogs. Biol. Neonat., 15: 54 (1968).

4. Burd, L. I., Jones, M. D., Simmons, M. A., Makowski, E. L., Meshcia, G., and Battaglia, F. C.: Placental production and fetal utilization of lactate and pyruvate. Nature (Lond.), 254: 710 (1975).

5. Downing, S. E., Lee, J. C., Taylor, J. F. N., and Halloran, K.: Influence of norepinephrine and digitalis on myocardial oxygen consumption in the newborn lamb. Circ. Res., 32: 471 (1973).

6. Fisher, D. J.: (unpublished observations)

7. Fisher, D. J., Heymann, M. A., and Rudolph, A. M.: Myocardial oxygen and carbohydrate consumption in fetal lambs in utero and in adult sheep. Am. J. Physiol., 238: H399 (1980).

8. Heymann, M. A., Paye, B. D., Hoffman, J. I. E. and Rudolph, A. M.: Blood flow measurement with radionuclide-labelled microspheres. Prog. Cardiovasc. Dis., 20: 55 (1977).

9. Kitamura, K., Jorgensen, C. R., Gobel, F. L., Taylor, H. L., and Wang, Y. Hemodynamic correlates of myocardial oxygen consumption during upright exercise. J. Appl. Physiol., 32: 516 (1972).

10. Klopfenstein, H. S., and Rudolph, A. M.: Postnatal changes in the circulation and responses to volume loading in sheep. Circ. Res., 42: 839 (1978).

11. Lister, G., Walter, T. K., Vermold, H. T., Dallman, P. R., and Rudolph, A. M. Oxygen delivery in lambs: cardiovascular and hematologic development. Am. J. Physiol., 237: H668 (1979).

12. May, N. D. S.: The anatomy of sheep. (University Queensland Press, St. Lucia, Brisbane. Queensland, 1964).

13. Opie, L. H.: Metabolism of the heart in health and disease. Part II. Am. Heart J. 77: 100 (1969).

14. Parmley, W. W., and Tyberg. J. V.: Determinants of myocardial oxygen demand. In: P. N. Yu, J. F. Goodwin: Progress in Cardiology. Vol. 5, pp. 19-36 (Philadelphia: Lea and Febiger, 1976).

15. Su, J. Y., and Friedman, W. F.: Comparison of the responses of fetal and adult cardiac muscle to hypoxia. Am. J. Physiol., 224: 1249 (1973).

16. Tsoulos, N. G., Colwill, J. R., Battaglia, F. C., Makowski, E. L., and Meschia. $\mathrm{G}$.: Comparisons of glucose, fructose, and $\mathrm{O}_{2}$ uptakes by fetuses of fed and starved ewes. Am. J. Physiol., 221: 234 (1971).

17. Van Duyne, C. M., Parker, H. R., Havel, R. J., and Holm, L. W.: Free fatty acid metabolism in fetal and newborn sheep. Am. J. Physiol., 199: 7 (1960).

18. Wittels, B., and Bressler, R.: Lipid metabolism in the newborn heart. J.C.I. 44: 1639 (1965).

19. Zar, J. H.: Biostatistical Analysis. (Englewood Cliffs, Prentice-Hall, Inc., 1974).

20. Zierler, K. L.: Free fatty acids as substrates for heart and skeletal muscle. Circ. Res., 38: 459 (1976)

21. Zivin, J. A., and Bartko, J. J.: Statistics for disinterested scientists. Life Sci., 18. 1526 (1974).

22. Requests for reprints should be addressed to: David J. Fisher, M.D., University of Texas Medical School at Houston, Department of Pediatrics, P. O. Box 20708, Houston, TX 77025 (USA).

23. This research was supported by a grant from the United States Public Health Service, Program Project Grant HL 06285. Dr. Fisher was a recipient of a National Research Service Award (SF HD 05483) from the National Institute of Child Health and Human Development.

24. Received for publication April 18, 1980.

25. Accepted for publication October 2, 1980. 